\title{
Surveillance of the Second Wave of COVID-19 in Europe: Longitudinal Trend Analyses
}

Lori Post ${ }^{1}$, PhD; Kasen Culler ${ }^{2}$, BSc; Charles B Moss ${ }^{3}$, PhD; Robert L Murphy ${ }^{4}$, MD; Chad J Achenbach ${ }^{5}$, MD, MPH; Michael G Ison ${ }^{5}$, MD, MSc; Danielle Resnick ${ }^{6}$, PhD; Lauren Nadya Singh ${ }^{1}$, MPH; Janine White ${ }^{1}$, MA; Michael J Boctor $^{2}$, BSc; Sarah B Welch ${ }^{1}$, MPH; James Francis Oehmke ${ }^{1}, \mathrm{PhD}$

\footnotetext{
${ }^{1}$ Buehler Center for Health Policy and Economics, Feinberg School of Medicine, Northwestern University, Chicago, IL, United States

${ }^{2}$ Feinberg School of Medicine, Northwestern University, Chicago, IL, United States

${ }^{3}$ Institute of Food and Agricultural Sciences, University of Florida, Gainsville, FL, United States

${ }^{4}$ Institute of Global Health, Feinberg School of Medicine, Northwestern University, Chicago, IL, United States

${ }^{5}$ Divison of Infectious Disease, Feinberg School of Medicine, Northwestern University, Chicago, IL, United States

${ }^{6}$ International Food Policy Research Institute, Washington DC, DC, United States
}

\section{Corresponding Author:}

Lori Post, PhD

Buehler Center for Health Policy and Economics

Feinberg School of Medicine

Northwestern University

420 E Superior

Chicago, IL, 60611

United States

Phone: 12039807107

Email: 1ori.post@northwestern.edu

\section{Abstract}

Background: The COVID-19 pandemic has severely impacted Europe, resulting in a high caseload and deaths that varied by country. The second wave of the COVID-19 pandemic has breached the borders of Europe. Public health surveillance is necessary to inform policy and guide leaders.

Objective: This study aimed to provide advanced surveillance metrics for COVID-19 transmission that account for weekly shifts in the pandemic, speed, acceleration, jerk, and persistence, to better understand countries at risk for explosive growth and those that are managing the pandemic effectively.

Methods: We performed a longitudinal trend analysis and extracted 62 days of COVID-19 data from public health registries. We used an empirical difference equation to measure the daily number of cases in Europe as a function of the prior number of cases, the level of testing, and weekly shift variables based on a dynamic panel model that was estimated using the generalized method of moments approach by implementing the Arellano-Bond estimator in R.

Results: New COVID-19 cases slightly decreased from 158,741 (week 1, January 4-10, 2021) to 152,064 (week 2, January 11-17, 2021), and cumulative cases increased from 22,507,271 (week 1) to 23,890,761 (week 2), with a weekly increase of 1,383,490 between January 10 and January 17. France, Germany, Italy, Spain, and the United Kingdom had the largest 7-day moving averages for new cases during week 1. During week 2, the 7-day moving average for France and Spain increased. From week 1 to week 2 , the speed decreased (37.72 to 33.02 per 100,000), acceleration decreased $(0.39$ to -0.16 per 100,000$)$, and jerk increased ( -1.30 to 1.37 per 100,000$)$.

Conclusions: The United Kingdom, Spain, and Portugal, in particular, are at risk for a rapid expansion in COVID-19 transmission. An examination of the European region suggests that there was a decrease in the COVID-19 caseload between January 4 and January 17, 2021. Unfortunately, the rates of jerk, which were negative for Europe at the beginning of the month, reversed course and became positive, despite decreases in speed and acceleration. Finally, the 7-day persistence rate was higher during week 2 than during week 1 . These measures indicate that the second wave of the pandemic may be subsiding, but some countries remain at risk for new outbreaks and increased transmission in the absence of rapid policy responses. 


\section{KEYWORDS}

SARS-CoV-2 surveillance; wave two; second wave; global COVID surveillance; Europe Public Health Surveillance; Europe COVID; Europe surveillance metrics; dynamic panel data; generalized method of the moments; Europe econometrics; Europe SARS-CoV-2; Europe COVID surveillance system; European COVID transmission speed; European COVID transmission acceleration; COVID transmission deceleration; COVID transmission jerk; COVID 7-day lag; SARS-CoV-2; Arellano-Bond estimator; GMM; Albania; Andorra; Austria; Belarus; Belgium; Bosnia and Herzegovina; Bulgaria; Croatia; Czech Republic; Denmark; Estonia; Finland; France; Germany; Greece; Greenland; Hungary; Iceland; Ireland; Isle of Man; Italy; Latvia; Liechtenstein; Lithuania; Luxembourg; Moldova; Monaco; Montenegro; Netherlands; Norway; Poland; Portugal; Romania; San Marino; Serbia; Slovakia; Slovenia; Spain; Sweden; Switzerland; Ukraine; United Kingdom; Vatican City

\section{Introduction}

\section{Background}

The first European COVID-19 case was reported on January 24, 2020, in France, with subsequent cases confirmed in Germany and Finland days later [1]. On March 11, 2020, the World Health Organization (WHO) declared that the spread of the novel coronavirus had exceeded the threshold of a pandemic [2] and, on March 13, 2020, the WHO declared Europe as the global epicenter, when their caseload and deaths exceeded the combined caseload in the rest of the world [1] (See Figure 1). The European Union (EU) closed all external borders on March 17, 2020 [1]. Although the EU coordinated the COVID-19 response between member countries, individual governments enacted separate national policies and made individual decisions regarding border closure and quarantine measures [3]. COVID-19 caseloads decreased for most European countries after peaking in April and May [4].

At present, European countries are experiencing a second wave of COVID-19 [5-11]. The WHO has warned that the death counts in Europe could surpass the peak observed in April 2020 [12]. Nations worldwide are struggling to control COVID-19 transmission by imposing social isolation and economic restrictions, with leaders reluctant to shut down businesses and quarantine citizens again [13,14]. As of February 9, 2021, the WHO reported 106,125,682 confirmed COVID-19 cases and 2,320,497 deaths worldwide [15]. Collectively, 33,534,153 COVID-19 cases have been reported in the EU and the United Kingdom, which have resulted in 740,733 deaths [4].

The World Bank (WB), a global partnership dedicated to reducing poverty and increasing sustainable prosperity in developing nations, divides the world into regions based on shared geographical, development, and cultural or historical features [16]. The Global SARS-CoV-2 Surveillance Project: Policy, Persistence, \& Transmission provides surveillance data [17] based on these WB-defined regions. The focus of this study is on the spread of COVID-19 specifically within the Western European region, including Albania, Andorra, Austria, Belarus, Belgium, Bosnia and Herzegovina, Bulgaria, Croatia, Czech Republic, Denmark, Estonia, Finland, France, Germany, Greece, Greenland, Hungary, Iceland, Ireland, Isle of Man, Italy, Latvia, Liechtenstein, Lithuania, Luxembourg, Moldova, Monaco, Montenegro, the Netherlands, Norway, Poland, Portugal, Romania, San Marino, Serbia, Slovakia, Slovenia, Spain, Sweden, Switzerland, Ukraine, the United Kingdom, and Vatican City.

Figure 1. Timeline of COVID-19-related events and decisions made (2020). EU: European Union; WHO: World Health Organization.

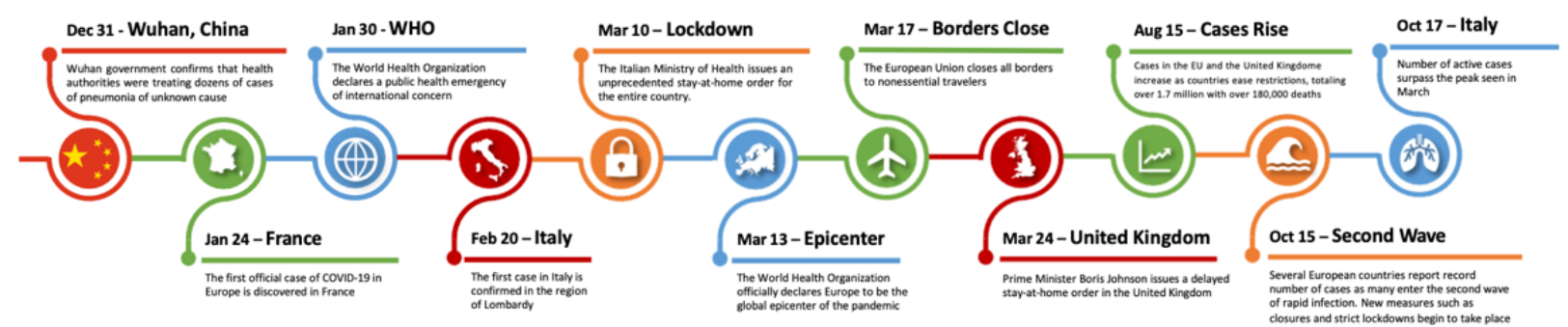

\section{Outbreak and Governance}

\section{Policies and Culture}

Analysis of COVID-19 cumulative incidence indicates that the drastic measures undertaken by the Italian government slowed the spread of the disease to lower than the expected 7-10 days after restrictions were implemented [18]. The rapid transmission was likely due to high population density [19], and the high case-fatality rate is associated with the older age distribution in Italy, wherein approximately $23 \%$ of the Italian population was aged 65 years or older in 2019 [20].

Other factors that influenced the severity of the COVID-19 were family structures, which likely increased interaction among family members [21]. Additionally, Southern European countries engage in physical greetings, with kisses on the cheek and friendly hugs being common in Italy, Spain, and France [22]. These cultural practices may be a contributing factor to the increased transmission of COVID-19 and related mortality in the Southern European countries listed above, where the virus spread very rapidly and yielded severe adverse effects [23].

In contrast, in Northern European countries such as Sweden, children tend to leave home earlier and frequently move farther away from their parents, often to pursue higher education. A "post-nuclear family structure" has developed more rapidly, and children in these countries may have less frequent contact with their families from an earlier age than those in the more traditional Southern European countries [21]. Additionally, 
personal space is valued to a higher degree, and kissing is less commonly used as a greeting compared to shaking hands or other less physical forms of greeting [24]. Sweden enacted less strict policies than Southern European countries did and saw similar results as countries that enacted late-onset stringent mandates [25]. It is worth noting that Sweden's per capita COVID-19 death rate far outpaces that of its Scandinavian neighbors, decreasing confidence in their mitigation strategies [26]. At the other extreme lie countries such as Hungary, where the Prime Minister pushed through legislation that allowed him to rule by decree for however long the pandemic continues and mandates jail time for the spread of disinformation, leading to concerns about restrictions on human rights and media freedoms [27].

The United Kingdom, physically and organizationally separated from its European neighbors since leaving the European Union, took a delayed and somewhat hesitant approach to controlling the spread of the virus. The first two COVID-19 cases in the nation were confirmed on January 31, 2020. The Department of Health and Social Care's coronavirus action plan was approved on March 3, 2020, outlining the country's plan to deploy four phased actions to deal with the pandemic: Contain, Delay, Research, and Mitigate [28]. The government moved from the Contain phase to the Delay phase on March 12, 2020 , after Italy had already locked down, and emphasized testing in hospital settings and not communities, with unrestricted entry to the country via ports and airports [28]. On March 19, 2020, COVID-19 was reclassified from level 4 to a milder threat level (ie, level 3) by the Advisory Committee on Dangerous Pathogens, allowing hospital infection control requirements to be lowered [28]. Finally, on March 24, 2020, the Prime Minister declared an enforceable lockdown across the nation [28], but COVID-19 spread rapidly throughout the United Kingdom, leading Europe in COVID-19-related deaths at over 43,579 [4]. Many European countries are experiencing a second wave of infections, with surging daily case numbers in France, Spain, the Netherlands, and the United Kingdom, and the WHO warning that, within the coming months, daily death counts could surpass the April peak observed in Europe [12]. National governments are struggling to control the infection due to increased pushback from local governments who are reluctant to shut down businesses and quarantine citizens a second time after being allowed to open up [13].

\section{Economics and Food Insecurity}

An important impact of the pandemic is the risk of food insecurity in vulnerable nations such as Ukraine and Moldova [29]. Much of the population in Ukraine lacks the ability to buy a sufficient amount of healthy food and often resides in conflict-affected areas of the country. Moreover, the current pandemic threatens to impact Ukraine's wheat exportation and livestock processing, which could create even more scarcity in affordable food for its citizens [30,31]. Ukraine responded with early restrictive policies in response to widespread fear among citizens, and the country ended up reporting fewer cases than Russia and Belarus, indicating that its response was most likely effective in slowing disease transmission [32]. However, economic growth in Ukraine was stable at $3.2 \%$ in 2019 , but the pandemic has forced a sudden slowdown in economic activity; the future of the economy will be dependent on the country's ability to support investment and diversify exports after the pandemic subsides [33].

Economic growth in Moldova had already declined sharply to $0.2 \%$ in late 2019 , and the unemployment rate saw an increase compared to 2018 [33]. Many citizens of Moldova rely heavily on food self-provisioning or food sharing within village networks [34]. Poverty is expected to increase in response to the COVID-19 pandemic, and the effects will likely impact households with inadequate insurance mechanisms. The maintenance of food security and economic stability will depend on the government's ability to alleviate food shortages and compensate for lost income, as well as to support jobs and growth when the crisis subsides [33].

\section{Surveillance}

Public health surveillance informs policy on "flattening the curve" of COVID-19 spread [17,35-37]. Epidemiologists have utilized various modeling techniques to forecast COVID-19 case numbers and attributed deaths [38-42]. The European Center for Disease Control, the WHO, and the Center for Systems Science and Engineering at Johns Hopkins University have developed tracking tools [11,38]. Although helpful, these static metrics are limited by incomplete case ascertainment and data contamination $[17,36]$. Existing surveillance is a proxy for the true COVID-19 caseload because public health surveillance systems tend to pick up the most severe cases [43,44], which is especially problematic when tracking SARS-CoV-2 infections because most carriers are asymptomatic or presymptomatic or may have mild symptoms [45-48]. Therefore, public health surveillance that can control for these limitations are needed. Moreover, metrics that detect the speed of transmission of the novel coronavirus, shifts in the pandemic, and acceleration of the speed and persistence of COVID-19 based on prior infections are needed to supplement existing measures.

\section{Significance}

Ideally, the development of a more advanced methodology for tracking and estimating COVID-19 transmission in regions within Europe will allow for a more reliable analysis of which policies are effective and what other factors may be associated with transmission rates. Public health departments, in addition to several universities and media outlets, are tracking COVID-19 metrics by using raw data, including the number of new cases, diagnostic tests, positive results, transmission rates and deaths, in addition to other measures such as local hospital capacity [4,49-57]. To remove temporal effects, many surveillance systems have shifted to 7-day moving averages to counter the dearth of reporting during holidays and weekends. Although moving averages temper volatility of data and testing or reporting affects, surveillance is still limited by missing cases. General public health surveillance is helpful and provides a proxy of the pandemic, but surveillance data are still limited by significant bias due to undercounts, reporting delays, testing errors, dearth of testing, asymptomatic carriers, and other types of data contamination. In fact, surveillance systems are predicated on the fact that they tend to include only the more severe cases, whereas mild cases and undiagnosed infections and deaths are excluded [43,44]. 
To that end, the objective of our study is to use a longitudinal trend analysis study design in concert with Dynamic Panel Modeling and Method of Moments to correct for existing surveillance data limitations [17,36]. Specifically, we will measure significant weekly shifts in the increase, decrease, or plateaued transmission of COVID-19. We will also measure the underlying causal effect from the previous week that persists through the current week, with a 7-day persistence rate to explain a clustering-declustering effect. The 7-day persistence represents an underlying disease transmission wave, wherein a large number of transmissions 7 days ago that resulted in a large number of infections today then echoes forward into a large number of new transmissions and, hence, a large number of new cases 7 days forward. An example of the 7-day lag would be large sporting events in the United Kingdom that drew huge crowds weekend after weekend even after new COVID-19 cases were confirmed in the country. Other potential "superspreader" events such as the exportation of COVID-19 cases from a popular ski town in the Austrian Alps back in March 2020 [58], would certainly contribute to this persistence effect as well. In summary, we will measure negative and positive shifts in the transmission of SARS-CoV-2 or the acceleration or deceleration rates. Our surveillance metric will provide public health surveillance data to inform governments in decision-making regarding disease control, mitigation strategies, and reopening policies as they continue to manage this unprecedented situation.

\section{Methods}

Our World in Data [59] compiles data from multiple sources on the web. Data for the most recent 7 weeks were accessed from the GitHub repository [60]. This resulted in a panel of 39 countries in Western Europe with 62 days in each panel $(n=2418)$. Based on published reports [16,61], an empirical difference equation was specified in which the number of new positive cases in each country at each day is a function of the prior number of cases, the level of testing, and weekly shift variables that measure whether the contagion was growing faster, at the same speed, or slower than in the previous weeks. This resulted in a dynamic panel model that was estimated using the generalized method of moments (GMM) approach by implementing the Arellano-Bond estimator in R [17,36].

\section{Results}

\section{Country Regression Results}

Regression results are presented for 39 European countries in Table 1. Weekly surveillance data presented in Tables 2-6 are based in part on these regressions. Data for 44 European countries were collected, but data for 5 countries were excluded in the regression analysis due to missing data. The regression Wald statistic is significant $\left(X_{8}^{2}=4980 ; P<.001\right)$. The Sargan test was not significant, failing to reject the validity of overidentifying restrictions $\left(X^{2}{ }_{511}=39 ; P=.39\right)$.

The coefficient for the 7-day lag was positive and statistically significant $(0.90, P<.001)$, indicating the number of infections 7 days prior to the study had a positive relationship that echoed forward 7 days later. The shift parameter 14 days ago was negative and statistically significant (coefficient $-0.30, P<.001$ ), suggesting that exogenous shift events had a negative effect on total case numbers (Table 1).

Table 1. Arellano-Bond dynamic panel data model of COVID-19 dynamics at the country level in Europe.

\begin{tabular}{lll}
\hline Variable & Coefficient & $P$ value \\
\hline 7-day lag & 0.90 & $<.001$ \\
Cumulative tests & -0.000 & .42 \\
7-day lag shift & -0.30 & $<.001$ \\
Weekend & -2.1 & .02 \\
\hline
\end{tabular}


Table 2. Static surveillance metrics for European countries for the week of January 4-10, 2021.

\begin{tabular}{|c|c|c|c|c|c|c|c|c|}
\hline Country & $\begin{array}{l}\text { New weekly } \\
\text { COVID-19 cas- } \\
\text { es }\end{array}$ & $\begin{array}{l}\text { Cumulative COVID- } \\
19 \text { cases }\end{array}$ & $\begin{array}{l}\text { 7-day mov- } \\
\text { ing average } \\
\text { of new } \\
\text { COVID-19 } \\
\text { cases }\end{array}$ & $\begin{array}{l}\text { Infection } \\
\text { rate per } \\
100,000 \text { pop- } \\
\text { ulation }\end{array}$ & $\begin{array}{l}\text { New week- } \\
\text { ly deaths }\end{array}$ & $\begin{array}{l}\text { Cumulative } \\
\text { deaths due to } \\
\text { COVID-19 }\end{array}$ & $\begin{array}{l}\text { 7-day moving av- } \\
\text { erage of new } \\
\text { COVID-19-relat- } \\
\text { ed deaths }\end{array}$ & $\begin{array}{l}\text { Deaths } \\
\text { rate per } \\
100,000 \\
\text { popula- } \\
\text { tion }\end{array}$ \\
\hline Albania & 562 & 63,595 & 593.86 & 19.53 & 8 & 1241 & 6.86 & 0.28 \\
\hline Andorra & 0 & 8586 & 56.29 & 0.00 & 0 & 85 & 0.14 & 0 \\
\hline Austria & 1651 & 380,722 & 2136.29 & 18.33 & 36 & 6723 & 57 & 0.40 \\
\hline Belarus & 1833 & 212,201 & 1748.43 & 19.40 & 10 & 1517 & 9.43 & 0.11 \\
\hline Belgium & 1569 & 664,263 & 2036 & 13.54 & 40 & 20,078 & 53.86 & 0.35 \\
\hline $\begin{array}{l}\text { Bosnia \& Herze- } \\
\text { govina }\end{array}$ & 254 & 115,633 & 426.86 & 7.74 & 25 & 4330 & 28.43 & 0.76 \\
\hline Bulgaria & 105 & 208,511 & 780 & 1.51 & 29 & 8126 & 64 & 0.42 \\
\hline Croatia & 646 & 219,993 & 1005 & 15.74 & 26 & 4368 & 42.29 & 0.63 \\
\hline Czech Republic & 8449 & 831,165 & $12,954.86$ & 78.90 & 137 & 13,115 & 165 & 1.28 \\
\hline Denmark & 1246 & 182,161 & 1829 & 21.51 & 28 & 1571 & 28.14 & 0.48 \\
\hline Estonia & 427 & 33,516 & 626.43 & 32.19 & 5 & 283 & 5.57 & 0.38 \\
\hline Finland & 198 & 38,590 & 259.71 & 3.57 & 0 & 586 & 3.57 & 0 \\
\hline France & 159,44 & $2,840,864$ & $18,269.86$ & 24.43 & 151 & 67,885 & 388.71 & 0.23 \\
\hline Germany & 948 & $1,929,410$ & $20,787.71$ & 1.13 & 339 & 40,936 & 877.86 & 0.40 \\
\hline Greece & 445 & 144,738 & 662.71 & 4.27 & 36 & 5263 & 43.71 & 0.35 \\
\hline Hungary & 1778 & 342,237 & 2034.57 & 18.41 & 94 & 10,648 & 109.14 & 0.97 \\
\hline Iceland & 10 & 5890 & 19.43 & 2.93 & 0 & 35 & 0 & 0 \\
\hline Ireland & 6886 & 147,613 & 6532.29 & 139.46 & 8 & 2344 & 12.14 & 0.16 \\
\hline Italy & 18,625 & $2,276,491$ & $17,292.14$ & 30.80 & 361 & 78,755 & 489.00 & 0.60 \\
\hline Latvia & 616 & 49,568 & 1010.14 & 32.66 & 31 & 849 & 24.14 & 1.64 \\
\hline Lithuania & 1492 & 159,672 & 1862.14 & 54.81 & 26 & 2197 & 79.14 & 0.96 \\
\hline Luxembourg & 0 & 47,744 & 189.86 & 0.00 & 0 & 527 & 4.57 & 0.00 \\
\hline Malta & 184 & 14,396 & 187.71 & 41.67 & 1 & 233 & 1.86 & 0.23 \\
\hline Moldova & 298 & 149,391 & 502.57 & 7.39 & 9 & 3139 & 14.57 & 0.22 \\
\hline Netherlands & 6655 & 885,098 & 7485.14 & 38.84 & 55 & 12,461 & 107.71 & 0.32 \\
\hline Norway & 555 & 55,474 & 679.71 & 10.24 & 0 & 472 & 5.14 & 0 \\
\hline Poland & 9133 & $1,385,522$ & 9565.71 & 24.13 & 178 & 31,189 & 295.71 & 0.47 \\
\hline Portugal & 7502 & 483,689 & 8062.14 & 73.57 & 102 & 7803 & 97.86 & 1.00 \\
\hline Romania & 3082 & 671,284 & 4407.86 & 16.02 & 62 & 16,654 & 96.43 & 0.32 \\
\hline San Marino & 0 & 2628 & 28.57 & 0.00 & 0 & 64 & 0.71 & 0 \\
\hline Serbia & 3564 & 359,689 & 2259.86 & 40.79 & 69 & 3582 & 36.71 & 0.79 \\
\hline Slovakia & 2973 & 208,209 & 2963.71 & 54.45 & 82 & 2919 & 85.86 & 1.50 \\
\hline Slovenia & 763 & 139,281 & 2027.86 & 36.70 & 25 & 2998 & 27.86 & 1.20 \\
\hline Spain & 0 & $2,050,360$ & $17,442.14$ & 0 & 0 & 51,874 & 148.14 & 0 \\
\hline Sweden & 0 & 489,471 & 7441.71 & 0 & 0 & 9433 & 100.86 & 0.00 \\
\hline Switzerland & 0 & 477,983 & 3669.57 & 0 & 14 & 8267 & 74.29 & 0.16 \\
\hline Ukraine & 5322 & $1,150,265$ & 6161.14 & 12.17 & 115 & 20,641 & 144.43 & 0.26 \\
\hline United Kingdom & 55,026 & $3,081,368$ & $59,809.86$ & 81.06 & 567 & 81,567 & 918.57 & 0.84 \\
\hline Europe & 158,741 & $22,507,271$ & $208,581.43$ & 26.52 & 2669 & 524,758 & 4649.43 & 0.45 \\
\hline
\end{tabular}


Table 3. Static surveillance metrics for European countries for the week of January 11-17, 2021.

\begin{tabular}{|c|c|c|c|c|c|c|c|c|}
\hline Country & $\begin{array}{l}\text { New } \\
\text { weekly } \\
\text { COVID- } \\
19 \text { cases }\end{array}$ & $\begin{array}{l}\text { Cumulative } \\
\text { COVID-19 cases }\end{array}$ & $\begin{array}{l}\text { 7-day moving } \\
\text { average of } \\
\text { new COVID- } \\
19 \text { cases }\end{array}$ & $\begin{array}{l}\text { Infection rate } \\
\text { per } 100,000 \\
\text { population }\end{array}$ & $\begin{array}{l}\text { New week- } \\
\text { ly deaths }\end{array}$ & $\begin{array}{l}\text { Cumulative } \\
\text { deaths due to } \\
\text { COVID-10 }\end{array}$ & $\begin{array}{l}\text { 7-day moving } \\
\text { average of new } \\
\text { COVID-19-re- } \\
\text { lated deaths }\end{array}$ & $\begin{array}{l}\text { Deaths } \\
\text { rate per } \\
100,000 \\
\text { popula- } \\
\text { tion }\end{array}$ \\
\hline Albania & 474 & 67,690 & 585 & 16.47 & 7 & 1277 & 5.14 & 0.24 \\
\hline Andorra & 45 & 9083 & 71 & 58.24 & 0 & 91 & 0.86 & 0 \\
\hline Austria & 1267 & 393,778 & 1865.14 & 14.07 & 29 & 7082 & 51.29 & 0.32 \\
\hline Belarus & 1924 & 22,5461 & 1894.29 & 20.36 & 9 & 1582 & 9.29 & 0.10 \\
\hline Belgium & 1630 & 678,839 & 2082.29 & 14.06 & 39 & 20,435 & 51 & 0.34 \\
\hline $\begin{array}{l}\text { Bosnia \& Herzegov- } \\
\text { ina }\end{array}$ & 0 & 11,7011 & 196.86 & 0 & 0 & 4411 & 11.57 & 0 \\
\hline Bulgaria & 77 & 211,813 & 471.71 & 1.11 & 9 & 8483 & 51 & 0.13 \\
\hline Croatia & 379 & 224,954 & 708.71 & 9.23 & 28 & 4616 & 35.43 & 0.68 \\
\hline Czech Republic & 5253 & 889,159 & 8284.86 & 49.05 & 123 & 14,338 & 174.71 & 1.15 \\
\hline Denmark & 889 & 189,767 & 1086.57 & 15.35 & 28 & 1776 & 29.29 & 0.48 \\
\hline Estonia & 388 & 37,079 & 509 & 29.25 & 5 & 325 & 6 & 0.38 \\
\hline Finland & 236 & 40,337 & 249.57 & 4.26 & 0 & 618 & 4.57 & 0 \\
\hline France & 37,405 & $2,969,091$ & $18,318.14$ & 57.31 & 329 & 70,422 & 362.43 & 0.50 \\
\hline Germany & 11,484 & $2,050,129$ & $17,245.57$ & 13.71 & 437 & 46,901 & 852.14 & 0.52 \\
\hline Greece & 237 & 148,607 & 552.71 & 2.27 & 28 & 5469 & 29.43 & 0.27 \\
\hline Hungary & 1241 & 351,828 & 1370.14 & 12.85 & 77 & 11,341 & 99 & 0.80 \\
\hline Iceland & 0 & 5956 & 9.43 & 0 & 0 & 35 & 0 & 0 \\
\hline Ireland & 2946 & 172,726 & 3587.57 & 59.66 & 13 & 2608 & 37.71 & 0.26 \\
\hline Italy & 125,44 & $2,381,277$ & $14,969.43$ & 20.75 & 377 & 82,177 & 488.86 & 0.62 \\
\hline Latvia & 567 & 55,664 & 870.86 & 30.06 & 17 & 978 & 18.43 & 0.90 \\
\hline Lithuania & 836 & 167,516 & 1120.57 & 30.71 & 31 & 2445 & 35.43 & 1.14 \\
\hline Luxembourg & 0 & 48,630 & 126.57 & 0 & 0 & 549 & 3.14 & 0 \\
\hline Malta & 141 & 15,588 & 170.29 & 31.93 & 1 & 239 & 0.86 & 0.23 \\
\hline Moldova & 214 & 152,854 & 494.71 & 5.30 & 5 & 3250 & 15.86 & 0.12 \\
\hline Netherlands & 5643 & 925,355 & 5751 & 32.93 & 41 & 13,107 & 92.29 & 0.24 \\
\hline Norway & 206 & 58,651 & 453.86 & 3.80 & 0 & 517 & 6.43 & 0.00 \\
\hline Poland & 5970 & $1,435,582$ & 7151.43 & 15.77 & 142 & 33,355 & 309.43 & 0.38 \\
\hline Portugal & 10,385 & 549,801 & 9444.57 & 101.85 & 152 & 8861 & 151.14 & 1.49 \\
\hline Romania & 2156 & 693,644 & 3194.29 & 11.21 & 57 & 17,221 & 81 & 0.30 \\
\hline San Marino & 0 & 2778 & 21.43 & 0 & 0 & 65 & 0.14 & 0 \\
\hline Serbia & 1317 & 372,533 & 1834.86 & 15.07 & 20 & 3750 & 24 & 0.23 \\
\hline Slovakia & 573 & 223,325 & 2159.43 & 10.50 & 57 & 3475 & 79.43 & 1.04 \\
\hline Slovenia & 569 & 149,125 & 1406.29 & 27.37 & 40 & 3180 & 26 & 1.92 \\
\hline Spain & 0 & $2,252,164$ & $28,829.14$ & 0 & 0 & 53,314 & 205.71 & 0 \\
\hline Sweden & 0 & 523,486 & 4859.29 & 0 & 0 & 10,323 & 127.14 & 0 \\
\hline Switzerland & 0 & 495,228 & 2463.57 & 0 & 7 & 8682 & 59.29 & 0.08 \\
\hline Ukraine & 6398 & $1,198,512$ & 6892.43 & 14.63 & 130 & 21,767 & 160.86 & 0.30 \\
\hline United Kingdom & 38,670 & $3,405,740$ & $46,338.86$ & 56.96 & 682 & 89,429 & 1123.14 & 1 \\
\hline Europe & 152,064 & $23,890,761$ & 17,7288 & 25.40 & 2920 & 558,494 & 4819.43 & 0.49 \\
\hline
\end{tabular}


Table 4. Novel surveillance metrics for European countries for the week of January 4-10, 2021.

\begin{tabular}{|c|c|c|c|c|}
\hline Country & $\begin{array}{l}\text { Speed }^{\mathrm{a}} \text { (weekly average of } \\
\text { new daily cases per } 100,000 \\
\text { population) }\end{array}$ & $\begin{array}{l}\text { Acceleration }{ }^{\mathrm{b}} \text { (weekly aver- } \\
\text { age, per } 100,000 \text { population) }\end{array}$ & $\begin{array}{l}\text { Jerk }^{\mathrm{c}} \text { (per } 100,000 \\
\text { population) }\end{array}$ & $\begin{array}{l}\text { 7-day persistence effect on } \\
\text { speed (number of new daily } \\
\text { cases per } 100,000 \text { popula- } \\
\text { tion attributed to new cases } \\
7 \text { days ago) }\end{array}$ \\
\hline Albania & 20.64 & 0.57 & 0.67 & 9.48 \\
\hline Andorra & 72.85 & -4.81 & -13.68 & 41.13 \\
\hline Austria & 23.72 & 0.29 & -1.11 & 13.20 \\
\hline Belarus & 18.50 & $-604,7296.00$ & 0.20 & 11.98 \\
\hline Belgium & 17.57 & 0.90 & -0.47 & 8.23 \\
\hline Bosnia \& Herzegovina & 13.01 & -1.08 & -3.08 & 7.71 \\
\hline Bulgaria & 11.23 & -0.14 & -0.25 & 6.58 \\
\hline Croatia & 24.48 & -0.17 & -4.16 & 16.75 \\
\hline Czech Republic & 120.97 & 4.58 & -8.31 & 55.90 \\
\hline Denmark & 31.58 & 0.17 & -0.15 & 22.78 \\
\hline Estonia & 47.22 & 0.92 & -0.90 & 24.14 \\
\hline Finland & 4.69 & 0.08 & -0.24 & 2.78 \\
\hline France & 27.99 & 0.75 & -2.90 & 12.66 \\
\hline Germany & 24.81 & -1.60 & -3.64 & 12.81 \\
\hline Greece & 6.36 & 0.08 & -0.66 & 3.82 \\
\hline Hungary & 21.06 & 0.70 & -1.23 & 10.58 \\
\hline Iceland & 5.69 & 0.42 & -0.13 & 1.78 \\
\hline Ireland & 132.29 & 5.57 & 1.38 & 27.34 \\
\hline Italy & 28.60 & 1.03 & -0.89 & 15.27 \\
\hline Latvia & 53.55 & 0.36 & -6.12 & 28.01 \\
\hline Lithuania & 68.40 & 1.33 & 0.57 & 50.47 \\
\hline Luxembourg & 30.33 & 0 & 0 & 16.50 \\
\hline Malta & 42.51 & 3.20 & -1.88 & 14.69 \\
\hline Moldova & 12.46 & 0.42 & 0.46 & 9.59 \\
\hline Netherlands & 43.68 & -0.67 & 0.38 & 29.34 \\
\hline Norway & 12.54 & 0.28 & 0.33 & 5.78 \\
\hline Poland & 25.27 & 1.26 & -0.15 & 13.75 \\
\hline Portugal & 79.07 & 5.77 & -2.97 & 27.46 \\
\hline Romania & 22.91 & 0.04 & -2.35 & 10.96 \\
\hline San Marino & 84.20 & 0 & 0 & 49.73 \\
\hline Serbia & 25.86 & 2.61 & 5.73 & 17.46 \\
\hline Slovakia & 54.28 & 4.59 & -1.52 & 31.29 \\
\hline Slovenia & 97.54 & 0.14 & -10.14 & 42.36 \\
\hline Spain & 37.31 & 0 & -203.00 & 13.43 \\
\hline Sweden & 73.69 & 0 & 0 & 35.06 \\
\hline Switzerland & 42.40 & 0 & 0 & 23.85 \\
\hline Ukraine & 14.09 & 0.14 & 0.23 & 9.96 \\
\hline United Kingdom & 88.10 & -0.03 & -0.50 & 46.37 \\
\hline Region & 37.72 & 0.39 & -1.30 & 18.97 \\
\hline
\end{tabular}


${ }^{a}$ Speed: Daily positive cases per 100,000 population.

${ }^{\mathrm{b}}$ Acceleration: day-to-day change in the number of positive cases per day.

${ }^{\mathrm{c}}$ Jerk: week-over-week change in acceleration. 
Table 5. Novel surveillance metrics for the week of January 11-17, 2021.

\begin{tabular}{|c|c|c|c|c|}
\hline Country & $\begin{array}{l}\text { Speed }^{\mathrm{a}} \text { (weekly average of } \\
\text { new daily cases per } 100,000 \\
\text { population) }\end{array}$ & $\begin{array}{l}\text { Acceleration }^{\mathrm{b}} \text { (weekly aver- } \\
\text { age per } 100,000 \text { population) }\end{array}$ & $\begin{array}{l}\text { Jerk }^{\mathrm{c}} \text { (per } 100,000 \\
\text { population) }\end{array}$ & $\begin{array}{l}\text { 7-day persistence effect on } \\
\text { speed (number of new daily } \\
\text { cases per } 100,000 \text { popula- } \\
\text { tion attributed to new cases } \\
7 \text { days ago) }\end{array}$ \\
\hline Albania & 20.33 & -0.44 & -0.07 & 12.37 \\
\hline Andorra & 91.89 & 8.32 & 9.24 & 43.68 \\
\hline Austria & 20.71 & -0.61 & 0.27 & 14.22 \\
\hline Belarus & 20.05 & 0.14 & -0.11 & 11.10 \\
\hline Belgium & 17.97 & 0.08 & -0.08 & 10.53 \\
\hline Bosnia \& Herzegovina & 6 & -1.11 & 0.89 & 7.80 \\
\hline Bulgaria & 6.79 & -0.06 & 0.27 & 6.73 \\
\hline Croatia & 17.26 & -0.93 & 1.29 & 14.68 \\
\hline Czech Republic & 77.36 & -4.26 & 0.82 & 72.54 \\
\hline Denmark & 18.76 & -0.88 & 1.25 & 18.93 \\
\hline Estonia & 38.37 & -0.42 & -0.88 & 28.32 \\
\hline Finland & 4.50 & 0.10 & 0.44 & 2.81 \\
\hline France & 28.06 & 4.70 & 9.05 & 16.78 \\
\hline Germany & 20.58 & 1.80 & 3.16 & 14.88 \\
\hline Greece & 5.30 & -0.29 & 0.11 & 3.81 \\
\hline Hungary & 14.18 & -0.79 & 1.10 & 12.63 \\
\hline Iceland & 2.76 & -0.42 & 0.13 & 3.41 \\
\hline Ireland & 72.66 & -11.40 & -6.74 & 79.33 \\
\hline Italy & 24.76 & -1.44 & -0.57 & 17.15 \\
\hline Latvia & 46.17 & -0.37 & 0.68 & 32.11 \\
\hline Lithuania & 41.16 & -3.44 & -0.71 & 41.02 \\
\hline Luxembourg & 20.22 & 0 & 0 & 18.19 \\
\hline Malta & 38.57 & -1.39 & 0.87 & 25.49 \\
\hline Moldova & 12.26 & -0.30 & -0.13 & 7.47 \\
\hline Netherlands & 33.56 & -0.84 & 0.87 & 26.19 \\
\hline Norway & 8.37 & -0.92 & -0.39 & 7.52 \\
\hline Poland & 18.90 & -1.19 & 0.11 & 15.16 \\
\hline Portugal & 92.62 & 4.04 & 1.98 & 47.41 \\
\hline Romania & 16.60 & -0.69 & 0.19 & 13.74 \\
\hline San Marino & 63.15 & 0 & -4.63 & 50.49 \\
\hline Serbia & 21 & -3.67 & -5.95 & 15.51 \\
\hline Slovakia & 39.55 & -6.28 & -0.98 & 32.55 \\
\hline Slovenia & 67.64 & -1.33 & 1.07 & 58.49 \\
\hline Spain & 61.66 & 0 & 0 & 22.37 \\
\hline Sweden & 48.12 & 0 & 0 & 44.18 \\
\hline Switzerland & 28.47 & 0 & 0 & 25.42 \\
\hline Ukraine & 15.76 & 0.35 & -0.63 & 8.45 \\
\hline United Kingdom & 68.26 & -3.44 & 0.49 & 52.83 \\
\hline Europe & 33.02 & -0.16 & 1.37 & 22.62 \\
\hline
\end{tabular}


${ }^{a}$ Speed: Daily positive cases per 100,000 population.

${ }^{\mathrm{b}}$ Acceleration: day-to-day change in the number of positive cases per day.

${ }^{\mathrm{c}}$ Jerk: week-over-week change in acceleration.

Table 6. Difference in 7-day persistence between the two study weeks.

\begin{tabular}{lllll}
\hline Rank & Week 1 (January 4-10, 2021) & Week 2 (January 11-17, 2021) & Difference \\
\hline 1 & Country & Difference & Country & 79.33 \\
2 & Czech Republic & 55.90 & Ireland & 72.54 \\
3 & Lithuania & 50.47 & Czech Republic & 58.49 \\
4 & San Marino & 49.73 & Slovenia & 52.83 \\
\hline
\end{tabular}

\section{Interpretation: Europe Regression Results}

The lagging indicators and shift parameters suggested recent changes in disease transmission in Europe between November 30, 2020, and January 17, 2021. The shift in the most recent 14 days, or 2 weeks, was negative and statistically significant $(P<.001)$. The coefficient for weekend was negative and statistically significant $(-2.1, P<.02)$, as shown in Table 1 .

\section{Surveillance Results}

Tables 2-6 display static and novel dynamic surveillance measures for the weeks of January 4-10, 2021, and January 11-17, 2021. Information pertaining to the prior weeks can be found in Tables S1-S8 of Multimedia Appendix 1. Static measures include the number of new cases during the first day of a given week, cumulative cases, the 7-day moving average of new cases, rate of infection, new deaths during the first day of a given week, cumulative deaths, the 7-day moving average of new deaths, and the rate of deaths (see Tables 2 and 3). The dynamic measures include a temporal element to better understand how past cases affect the present ones and how present cases affect the future ones. Dynamic measures (see Tables 4 and 5) include (1) speed-the number of new observed COVID-19 cases per day per 100,000, averaged over a week; (2) acceleration - the change in speed from the prior week to the current week; (3) jerk-the week-over-week change in acceleration as a function of time over the course of 2 weeks between January 4 and 17, 2021; and (4) the 7-day persistence effect on speed-the average of the number of new cases per day in a given week that are statistically attributable to new cases reported 7 days earlier.

Static measures in Europe for the week of January 4-10, 2021, are presented in Table 2 and those for the week of January 11-17, 2021, are presented in Table 3. New European cases slightly decreased from 158,741 to 152,064 during the first day of each week, with only cumulative cases increasing from $22,507,271$ to $23,890,761$, which is a weekly increase of 1,383,490 from January 10 to January 17, 2021. Cumulative deaths due to COVID-19 in Europe reached 558,494 by January 17, 2021. The 7-day moving average of new cases totaled 208,581 in the first week and 177,288 in the second week.

France, Germany, Italy, Spain, and the United Kingdom had the largest 7-day moving averages for new cases of infection at $18,269,20,787,17,292,17,442$, and 59,809 during the week of
January 4-10, 2021. In the second week (January 11-17, 2021), 7-day moving averages increased to 18,318 and 28,829 for France and Spain, respectively. The 7-day moving average for Germany, Italy, and the United Kingdom decreased to 17,245, 14,969 , and 46,338, respectively. The infection rate per 100,000 people during the week of January 4-10, 2021, was the highest in Ireland and the United Kingdom at 139.5 and 81.06, respectively. The Czech Republic, Portugal, and Lithuania reported the next highest rates at 78.90, 73.59, and 54.81 per 100,000 population. These 5 countries with the highest infection rates reported a change for the week of January 11-17, 2021, thereby also changing the ranking and magnitude of rates. The top 5 countries by infection rate in week 2 were Portugal at 101.85, Ireland at 59.66, Andorra at 58.24, France at 57.31, and the United Kingdom at 56.94 per 100,000 population.

During the week of January 4-10, 2021, the highest death rates were reported in Latvia, Slovakia, and the Czech Republic at $1.64,1.50$, and 1.28 per 100,000 population. The following week, the European countries with the highest death rates were Slovenia, Portugal, and the Czech Republic at 1.92, 1.49 and 1.15 per 100,000 population.

Tables 4 and 5 and Figure 2 (data sourced from The Global SARS-CoV-2 Surveillance Project [62]) display the dynamic metrics that offer a more temporal view of these data. Novel metrics are also displayed in Multimedia Appendices 2-4. From the week of January 4-10 to the week of January 11-17, 2021, in Europe, the dynamic measures of speed decreased (37.72 to 33.02 per 100,000$)$, acceleration decreased $(0.39$ to -0.16 per $100,000)$, and jerk increased $(-1.30$ to 1.37 per 100,000$)$. Speed was the highest and decreasing in Ireland (132.29 to 72.66 per 100,000), the Czech Republic (120.97 to 77.36 per 100,000), and Slovenia (97.54 to 67.64 per 100,000) during both weeks. Acceleration was the highest in Portugal, Ireland, and Slovakia in the week of January 4-10, 2021, at 5.77, 5.57, and 4.59 per 100,000 population, respectively. Only Ireland had a positive jerk during this time. Andorra, France, and Portugal had the largest acceleration rates during the week of January 4-10, with reported increases to $8.32,4.70$, and 4.04 per 100,000 population, respectively. Jerk was the highest in Serbia, Ireland, and Albania during the week of January 4-10, 2021, at 5.73, 1.38 , and 0.67 per 100,000 population. Andorra, France, and Germany reported the highest jerk rates per 100,000 in the week January 11-17, 2021, at 9.24, 9.05, and 3.16, respectively. 
Figure 2. Weekly SARS-CoV-2 trends by country in Europe (December 7, 2020, to January 17, 2021; data source: [62]).

\section{Country Weekly Statistics Trends Last 8 Weeks}

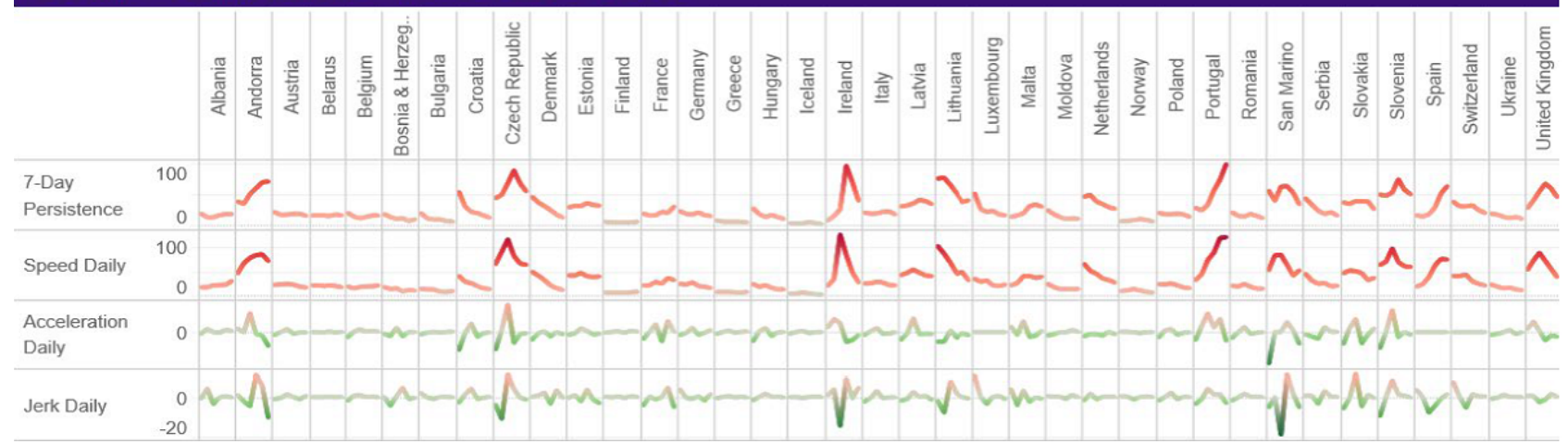

The 7-day persistence difference in Table 6 demonstrates the changes in 7-day persistence for the top 4 European countries between January 4-10 and January 11-17, 2021, suggesting an underlying shift that significantly increased persistence for some countries but significantly decreased persistence for other countries during the week of January 11-17. Only two of the countries were in the top 4 for both weeks (ie, the Czech Republic and United Kingdom). Lithuania and San Marino were in the top 4 for the first week, but Lithuania decreased to 41.01

Table 7. Most populous European countries.

\begin{tabular}{ll}
\hline Country & Population as of 2020 \\
\hline Germany & $83,783,942$ \\
United Kingdom & $67,886,011$ \\
France & $65,273,511$ \\
Italy & $60,461,826$ \\
Spain & $46,754,778$ \\
\hline
\end{tabular}

\section{Discussion}

\section{Principal Results}

Thus far, European COVID-19 infection surveillance has depended on static metrics with limited insight into longitudinal pandemic progression. Dynamic metrics provide an additional lens for surveillance that better captures the evolving prevalence of disease. After combining static and dynamic metrics, some European countries stand out as with the highest risk for uncontrolled growth. These high-risk countries must maintain transmission mitigating policies if they are to protect their citizens and the citizens of neighboring countries.

Europe, as a region, is still experiencing high COVID-19 case rates, but these appear to be trending downward as the region emerges from its second wave. The 7-day moving average of new cases showed a substantial decrease from 208,581 to 177,288 between January 4 and January 17, 2021. The 7-day moving average of COVID-19-related deaths, however, increased from the week of January 4-10 to the week of January 11-17, 2021. Speed of transmission in the region decreased and acceleration shifted from positive to negative from week 1 to week 2, suggesting that case rates may continue to trend downward in coming weeks. This shift in acceleration implies in the second week. San Marino slightly increased from 49.73 to 50.49 , but did not make it into the top 4 for the second week.

Among the top 5 countries by population (Table 7), Germany and France remained relatively stable, and the United Kingdom had the highest indicators of cause for alarm, with positive increases in speed and persistence but slightly negative decreases in acceleration and jerk. Smaller countries such as the Czech Republic, Ireland, Andorra, and Portugal reported higher positive increases in speed, acceleration, jerk, and persistence. that the speed was increasing at the beginning of the study period but entered a downward trajectory by the end. However, jerk shifted from negative to positive during these two weeks, indicating that the downward trend in acceleration was slowing toward the end of the study period. Interventions and continued precautions will be necessary to maintain a decreasing 7-day moving average of new cases and to continue the downward trajectory of speed and acceleration.

Infection rates show the countries that were the hardest hit at the time of data collection. The top 5 most populous countries in Europe are Germany, the United Kingdom, France, Italy, and Spain. Unsurprisingly, these 5 countries also had the largest 7-day moving averages for new infections during the study period. The United Kingdom had the second highest infection rate per 100,000 people during week 1 of the data collection, along with the largest 7-day moving average of new cases. This finding indicates that the United Kingdom may be at risk of increasing transmission, but the infection rate per 100,000 people decreased from 81.06 during week 1 to 56.94 in week 2 , which is reassuring. Both the speed of virus transmission and acceleration in the United Kingdom decreased over the recorded period as well, but jerk actually increased from -0.49 to 0.50 , and the country's 7-day persistence was the fourth highest in Europe during both weeks, indicating that the United Kingdom 
does need to stay vigilant and ensure proper enforcement of policies to reduce transmission in order to avoid another outbreak.

France and Germany both reported increases in acceleration over the two weeks, and the jerk transitioned from a negative value to a positive value, putting both countries at risk of experiencing increased growth in the coming weeks. Additionally, Spain had increasing speed and jerk, and its 7-day persistence effect increased over the 2-week period, indicating an increase in forward echoes of COVID-19 cases present in the country. Fortunately, Italy's speed and acceleration both decreased over the recorded period, and the jerk was negative during both weeks, implying that mitigation strategies are currently proving to be effective in Italy-the country that was initially one of the hardest hit. These 5 most populous countries are responsible for a very significant portion of the total cases in the European region, and they will likely require regional policy coordination for optimal control of virus transmission.

Some smaller countries in the region have also demonstrated dynamic metrics that warrant concern, such as Andorra. The speed increased from week 1 to week 2 , and jerk and acceleration both dramatically increased from negative to positive values, indicating that more intense restrictions are likely necessary to slow the spread. Ireland had the highest infection rate per 100,000 people during week 1 of data collection and the second highest infection rate during week 2 . Additionally, Ireland had the largest speed and jerk and the second largest acceleration during week 1, thereby increasing concern for a potential future outbreak in the country. However, all of these dynamic metrics decreased dramatically during week 2 (January 11-17, 2021), with acceleration and jerk actually transitioning to negative values, supporting the idea that Ireland's mitigation strategy is proving to be effective, at least during the time period in question.

Portugal was also at high risk of increased transmission, with a transmission rate per 100,000 people in the top 5 countries of the region during both weeks. With respect to novel dynamic metrics, Portugal had the largest positive acceleration in the region during week 1 and the third largest in week 2 . Additionally, the country's jerk increased from a negative value to a positive value and the 7-day persistence effect almost doubled from week to week. This finding indicates that Portugal should consider implementing new policies to reduce transmission and specifically to restrict the evolution of superspreader events, given the increase in 7-day persistence and the fact that Portugal had the second highest death rate per 100,000 people in the region during week 2 (January 11-17, 2021). Residents of Portugal were not only highly likely to contract COVID-19 during this time period, but they were also more likely to die of the disease than residents of most other European countries.

Although some European countries showed signs of uncontrolled growth for the near future, many demonstrate decreasing dynamic metrics that provide reassurance that transmission is being controlled appropriately. However, based on these results, countries with increasing dynamic metrics that are most at risk of outbreaks include Andorra, Portugal, and Spain. Fortunately,
Andorra's population is relatively small for the region, potentially insulating regional policy makers and agencies from an overwhelming surge in COVID-19 cases. In contrast, Spain and Portugal are relatively large countries. Their caseloads and positive dynamic metrics suggest that these two countries would require substantial effort to control the COVID-19 spread. Regional coordination would be essential given the size of these countries from a population and economic perspective. Additionally, some countries such as the Czech Republic have very high 7-day persistence effects but decreasing speed and acceleration, indicating that the overall transmission in the country may be decreasing, but focused policy targeted toward preventing superspreader events may be helpful.

Europe experienced a surge in COVID-19 transmission due to the second wave of the pandemic [11,63-65]. Because infection rates had significantly increased across Europe, many governments imposed strict lockdowns shutting down European economies again. Since SARS-CoV-2 cases were first reported in Europe earlier in 2020, COVID-19-related research has kept pace and, consequently, fewer deaths have been reported [61]. The virus is still just as contagious and deadly, but targeted therapies have resulted in attenuation of death rates across countries [61].

\section{Limitations}

Data are limited by granularity and collection method. Data were collected at the country level, which precludes local analysis of surveillance trends. Moreover, data collection mechanisms differ by country and may even differ by region within a given country. These different methods lead to weekend effects, missing data points, and other contamination.

\section{Comparison With Prior Work}

This study is part of a broader research program at Northwestern Feinberg School of Medicine, The Global SARS-CoV-2 Surveillance Project: Policy, Persistence, \& Transmission. This research program developed novel surveillance metrics to include speed, acceleration, jerk, and 7-day persistence at the country level $[17,66]$. We have also derived surveillance metrics for all global regions.

\section{Conclusion}

Static and dynamic public health surveillance tools provide a more complete picture of the progression of the COVID-19 pandemic across countries and regions. Although static measures, including infection rates and death rates, capture data at a given point in time, they are less successful in assessing population health over a period of weeks or months. By including speed, acceleration, jerk, and 7-day persistence, public health officials may design policies with an eye to the future. According to surveillance data, all countries in Europe that were at the highest risk during the second wave of the COVID-19 pandemic shared a number of characteristics. The United Kingdom, Spain, and Portugal demonstrated high infection rates, jerk, and 7-day persistence rates. Looking ahead, policy makers in these countries and the region at large should be concerned about growth in the already substantial number of COVID-19 cases over the short term. Given the substantial 7-day persistence rates in large countries such as the United 
Kingdom, Spain, and the Czech Republic, it is imperative that efforts be made to target superspreader events. Analysis of subsequent surveillance data using both static and dynamic tools can help confirm the efficaciousness of new policies.

\section{Acknowledgments}

This study was partially supported by Feed the Future through the United States Agency for International Development, under the terms of Contract No. 7200LA1800003. The opinions expressed herein are those of the authors and do not necessarily reflect the views of the United States Agency for International Development.

\section{Conflicts of Interest}

None declared.

\section{Multimedia Appendix 1}

Tables S1-S10. Static and novel surveillance metrics for specific weeks.

[DOCX File, 54 KB-Multimedia Appendix 1]

\section{Multimedia Appendix 2}

Map showing global weekly explosive growth potential of COVID-19 cases.

[PNG File , $511 \mathrm{~KB}$-Multimedia Appendix 2]

\section{Multimedia Appendix 3}

Map showing weekly speed of virus transmission by country in Europe.

[PNG File , 689 KB-Multimedia Appendix 3]

\section{Multimedia Appendix 4}

Map showing weekly acceleration/jerk by country in Europe.

[PNG File, 807 KB-Multimedia Appendix 4]

\section{References}

1. Linka K, Peirlinck M, Sahli Costabal F, Kuhl E. Outbreak dynamics of COVID-19 in Europe and the effect of travel restrictions. Comput Methods Biomech Biomed Engin 2020 Aug;23(11):710-717 [FREE Full text] [doi: 10.1080/10255842.2020.1759560] [Medline: 32367739]

2. Cucinotta D, Vanelli M. WHO Declares COVID-19 a Pandemic. Acta Biomed 2020 Mar 19;91(1):157-160 [FREE Full text] [doi: 10.23750/abm.v91i1.9397] [Medline: 32191675]

3. Goniewicz K, Khorram-Manesh A, Hertelendy AJ, Goniewicz M, Naylor K, Burkle FM. Current response and management decisions of the European Union to the COVID-19 outbreak: A review. Sustainability 2020 May 08;12(9):3838. [doi: $10.3390 /$ su12093838]

4. Weekly COVID-19 country overview. European Centre for Disease Prevention and Control. URL: https:/ /covid19-country-overviews.ecdc.europa.eu/ [accessed 2021-02-07]

5. Wise J. Covid-19: Risk of second wave is very real, say researchers. BMJ 2020 Jun 09;369:m2294. [doi: 10.1136/bmj.m2294] [Medline: 32518177]

6. Middleton J, Lopes H, Michelson K, Reid J. Planning for a second wave pandemic of COVID-19 and planning for winter: A statement from the Association of Schools of Public Health in the European Region. Int J Public Health 2020 Dec;65(9):1525-1527 [FREE Full text] [doi: 10.1007/s00038-020-01455-7] [Medline: 32857238]

7. Cacciapaglia G, Cot C, Sannino F. Second wave COVID-19 pandemics in Europe: a temporal playbook. Sci Rep 2020 Sep 23;10(1):15514 [FREE Full text] [doi: 10.1038/s41598-020-72611-5] [Medline: $\underline{\text { 32968181] }}$

8. Grech V, Cuschieri S. Withdrawn: COVID-19: A global and continental overview of the second wave and its (relatively) attenuated case fatality ratio. Early Hum Dev 2020 Oct 03:105211 [FREE Full text] [doi: 10.1016/j.earlhumdev.2020.105211] [Medline: 33039260]

9. Seligmann H, Iggui S, Rachdi M, Vuillerme N, Demongeot J. Inverted covariate effects for first versus mutated second wave Covid-19: High temperature spread biased for young. Biology (Basel) 2020 Aug 14;9(8):226 [FREE Full text] [doi: 10.3390/biology9080226] [Medline: 32823981]

10. Aleta A, Martín-Corral D, Piontti APY, Ajelli M, Litvinova M, Chinazzi M, et al. Modeling the impact of social distancing, testing, contact tracing and household quarantine on second-wave scenarios of the COVID-19 epidemic. medRxiv. Preprint posted online on May 18, 2020. [FREE Full text] [doi: 10.1101/2020.05.06.20092841] [Medline: $\underline{\text { 32511536] }}$ 
11. Win A. Rapid rise of COVID-19 second wave in Myanmar and implications for the Western Pacific region. QJM 2020 Dec 01;113(12):856-857 [FREE Full text] [doi: 10.1093/qjmed/hcaa290] [Medline: 33095232]

12. Reynolds E, Lister T. European nations smash Covid-19 records as WHO warns daily deaths could surpass April peak. CNN. URL: https://www.cnn.com/2020/10/16/europe/europe-coronavirus-records-intl/index.html [accessed 2021-04-09]

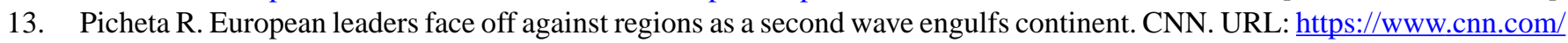
2020/10/18/europe/europe-coronavirus-restrictions-regions-intl/index.html [accessed 2021-04-09]

14. Stefana A, Youngstrom EA, Hopwood CJ, Dakanalis A. The COVID-19 pandemic brings a second wave of social isolation and disrupted services. Eur Arch Psychiatry Clin Neurosci 2020 Sep;270(6):785-786 [FREE Full text] [doi: 10.1007/s00406-020-01137-8] [Medline: $\underline{\text { 32415510] }}$

15. WHO Coronavirus (COVID-19) Dashboard. World Health Organization. URL: https://covid19.who.int/ [accessed 2021-02-03]

16. Kapur D, Lewis JP, Webb RC. The World Bank: Its First Half Century. Washington DC: Brookings Institution Press; 2011.

17. Oehmke JF, Moss CB, Singh LN, Oehmke TB, Post LA. Dynamic Panel Surveillance of COVID-19 Transmission in the United States to Inform Health Policy: Observational Statistical Study. J Med Internet Res 2020 Oct 5;22(10):e21955 [FREE Full text] [doi: 10.2196/21955] [Medline: $\underline{\text { 32924962] }}$

18. Sebastiani G, Massa M, Riboli E. Covid-19 epidemic in Italy: evolution, projections and impact of government measures. Eur J Epidemiol 2020 Apr;35(4):341-345 [FREE Full text] [doi: 10.1007/s10654-020-00631-6] [Medline: 32306149]

19. Filippi AR, Russi E, Magrini SM, Corvò R. Letter from Italy: First practical indications for radiation therapy departments during COVID-19 outbreak. Int J Radiat Oncol Biol Phys 2020 Jul 01;107(3):597-599 [FREE Full text] [doi: 10.1016/j.ijrobp.2020.03.007] [Medline: 32199941]

20. Onder G, Rezza G, Brusaferro S. Case-Fatality Rate and Characteristics of Patients Dying in Relation to COVID-19 in Italy. JAMA 2020 May 12;323(18):1775-1776. [doi: 10.1001/jama.2020.4683] [Medline: 32203977]

21. Bordone V. Contact and proximity of older people to their adult children: a comparison between Italy and Sweden. Popul. Space Place 2009 Jul;15(4):359-380. [doi: 10.1002/psp.559]

22. Fernandez J. Cross-cultural ethnology of greeting observances: From hands-free to hug-full. Legacy Volume Eight - Reading Area Community College. 2009 May. URL: https://www.racc.edu/sites/default/files/imported/StudentLife/Clubs/Legacy/ pdf/LegacyVIII.pdf\#page $=82$ [accessed 2020-11-14]

23. Ceylan Z. Estimation of COVID-19 prevalence in Italy, Spain, and France. Sci Total Environ 2020 Aug 10;729:138817 [FREE Full text] [doi: 10.1016/j.scitotenv.2020.138817] [Medline: 32360907]

24. Scroope C. Swedish Culture. The Cultural Atlas. 2017. URL: https://culturalatlas.sbs.com.au/swedish-culture [accessed 2020-11-14]

25. Kamerlin SCL, Kasson PM. anaging COVID-19 spread with voluntary public-health measures: Sweden as a case study for pandemic control. Clin Infect Dis 2020 Dec 15;71(12):3174-3181 [FREE Full text] [doi: 10.1093/cid/ciaa864] [Medline: 32609825]

26. Habib H. Has Sweden's controversial covid-19 strategy been successful? BMJ 2020 Jun 12;369:m2376. [doi: 10.1136/bmj.m2376] [Medline: 32532807]

27. Resnick D. Trust in science and in government plays a crucial role in COVID-19 response. International Food Policy Research Institute Blog. URL: https://www.ifpri.org/blog/trust-science-and-government-plays-crucial-role-covid-19-response [accessed 2021-04-13]

28. Scally G, Jacobson B, Abbasi K. The UK's public health response to covid-19. BMJ 2020 May 15;369:m1932. [doi: 10.1136/bmj.m1932] [Medline: 32414712]

29. Swinnen J, McDermott J, editors. COVID-19 and global food security. Washington, DC: International Food Policy Research Institute (IFPRI); 2020.

30. Klimova I. Food security in Ukraine and the world during a pandemic. Economics Management Innovations 2020;26(1):1-11 [FREE Full text] [doi: 10.35433/ISSN2410-3748-2020-1(26)-3]

31. Food Assistance Fact Sheet - Ukraine. USAID.gov. URL: https://www.usaid.gov/ukraine/food-assistance [accessed 2020-08-03]

32. Åslund A. Responses to the COVID-19 crisis in Russia, Ukraine, and Belarus. Eurasian Geography and Economics 2020 Jun 16;61(4-5):532-545. [doi: 10.1080/15387216.2020.1778499]

33. Europe and Central Asia Economic Update, Spring 2020: Fighting COVID-19. World Bank Group - Open Knowledge Repository. 2020. URL: https://openknowledge.worldbank.org/handle/10986/33476 [accessed 2020-08-03]

34. Piras S. Home - grown food and the benefits of sharing: The "intergenerational pact" in postsocialist Moldova. J Agrar Change 2019 Dec 20;20(3):460-484. [doi: 10.1111/joac.12351]

35. Ibrahim NK. Epidemiologic surveillance for controlling Covid-19 pandemic: types, challenges and implications. J Infect Public Health 2020 Nov;13(11):1630-1638 [FREE Full text] [doi: 10.1016/j.jiph.2020.07.019] [Medline: 32855090]

36. Oehmke JF, Oehmke TB, Singh LN, Post LA. Dynamic panel estimate-based health surveillance of SARS-CoV-2 infection rates to inform public health policy: Model development and validation. J Med Internet Res 2020 Sep 22;22(9):e20924 [FREE Full text] [doi: 10.2196/20924] [Medline: $\underline{\text { 32915762] }}$ 
37. Foddai A, Lubroth J, Ellis-Iversen J. Base protocol for real time active random surveillance of coronavirus disease (COVID-19) - Adapting veterinary methodology to public health. One Health 2020 Jun;9:100129 [FREE Full text] [doi: 10.1016/j.onehlt.2020.100129] [Medline: $\underline{32292815]}$

38. Dong E, Du H, Gardner L. An interactive web-based dashboard to track COVID-19 in real time. The Lancet Infectious Diseases 2020 May;20(5):533-534. [doi: 10.1016/s1473-3099(20)30120-1]

39. Lin Y, Liu C, Chiu Y. Google searches for the keywords of "wash hands" predict the speed of national spread of COVID-19 outbreak among 21 countries. Brain Behav Immun 2020 Jul;87:30-32 [FREE Full text] [doi: 10.1016/j.bbi.2020.04.020] [Medline: $\underline{\text { 32283286] }}$

40. Sajadi MM, Habibzadeh P, Vintzileos A, Shokouhi S, Miralles-Wilhelm F, Amoroso A. Temperature, humidity and latitude analysis to predict potential spread and seasonality for COVID-19. SSRN 2020 Mar 09:3550308. [doi: 10.2139/ssrn.3550308] [Medline: 32714105]

41. Hamzah FAB, Lau CH, Nazri H, Ligot DV, Lee G, Tan CL, et al. CoronaTracker: worldwide COVID-19 outbreak data analysis and prediction. Bull World Health Organ. Preprint posted online on March 19, 2020. [FREE Full text]

42. Petropoulos F, Makridakis S. Forecasting the novel coronavirus COVID-19. PLoS One 2020;15(3):e0231236 [FREE Full text] [doi: 10.1371/journal.pone.0231236] [Medline: 32231392]

43. Thacker SB, Berkelman RL. Public health surveillance in the United States. Epidemiol Rev 1988;10:164-190. [doi: 10.1093/oxfordjournals.epirev.a036021] [Medline: 3066626]

44. Teutsch SM. Considerations in planning a surveillance system. In: Principles \& Practice of Public Health Surveillance. Online: Oxford Scholarship; Sep 2010:18-28.

45. Day M. Covid-19: four fifths of cases are asymptomatic, China figures indicate. BMJ 2020 Apr 02;369:m1375. [doi: 10.1136/bmj.m1375] [Medline: $\underline{32241884]}$

46. He J, Guo Y, Mao R, Zhang J. Proportion of asymptomatic coronavirus disease 2019: A systematic review and meta-analysis. J Med Virol 2021 Feb;93(2):820-830 [FREE Full text] [doi: 10.1002/jmv.26326] [Medline: 32691881]

47. Al-Sadeq DW, Nasrallah GK. The incidence of the novel coronavirus SARS-CoV-2 among asymptomatic patients: A systematic review. Int J Infect Dis 2020 Sep;98:372-380 [FREE Full text] [doi: 10.1016/j.ijid.2020.06.098] [Medline: $\underline{32623083]}$

48. An P, Song P, Wang Y, Liu B. Asymptomatic patients with novel coronavirus disease (COVID-19). Balkan Med J 2020 Jun 01;37(4):229-230 [FREE Full text] [doi: 10.4274/balkanmedj.galenos.2020.2020.4.20] [Medline: 32279479]

49. Daily Summary - Coronavirus (COVID-19) in the UK. Gov.UK. URL: https://coronavirus.data.gov.uk/ [accessed 2020-08-16]

50. About CoronaTracker. CoronaTracker.com. URL: https://www.coronatracker.com/about [accessed 2020-08-16]

51. Coronavirus in Ukraine. Webpage in Ukrainian. Ministry of Health of Ukraine. URL: https://covid19.gov.ua [accessed 2020-08-16]

52. Guardia AB, Pawelec H, Hirsch C. Coronavirus in Europe: Live data tracker. Politico. 2020 Mar 13. URL: https://www. politico.eu/article/coronavirus-in-europe-by-the-numbers/ [accessed 2020-08-16]

53. Covid map: Coronavirus cases, deaths, vaccinations by country. BBC News. URL: https://www.bbc.com/news/ world-51235105 [accessed 2020-08-16]

54. Coronavirus tracker: the latest figures as countries fight the Covid-19 resurgence. The Financial Times. URL: https://www. ft.com/content/a2901ce8-5eb7-4633-b89c-cbdf5b386938 [accessed 2020-08-16]

55. Coronavirus Disease 2019 (COVID-19) - Situation Report of the Robert Koch Institute. Robert Koch Institute. URL: https:/ /www.rki.de/EN/Content/infections/epidemiology/outbreaks/COVID-19/Situationsberichte_Tab.html [accessed 2020-08-16]

56. Daily Situation Report. Webpage in Italian. Ministry of Health of Italy. URL: http://www.salute.gov.it/portale/ nuovocoronavirus/homeNuovoCoronavirus.jsp? [accessed 2020-08-16]

57. Situación actual. Ministerio de Sanidad, Consumo y Bienestar Social. URL: https://www.mscbs.gob.es/en/profesionales/ saludPublica/ccayes/alertasActual/nCov-China/situacionActual.htm [accessed 2020-08-16]

58. Correa-Martínez CL, Kampmeier S, Kümpers P, Schwierzeck V, Hennies M, Hafezi W, et al. A pandemic in times of global tourism: Superspreading and exportation of COVID-19 cases from a ski area in Austria. J Clin Microbiol 2020 May 26;58(6). [doi: $10.1128 / \mathrm{jcm} .00588-20]$

59. Ritchie H, Ortiz-Ospina E, Beltekian D, Mathieu E, Hasell J, Macdonald B, et al. Coronavirus Pandemic (COVID-19) Statistics and Research. Our World in Data. URL: https://ourworldindata.org/coronavirus [accessed 2021-10-19]

60. Covid-19-data. URL: https://github.com/owid/covid-19-data [accessed 2021-04-13]

61. Kottasova C. Covid-19 deaths aren't rising as fast in Europe and US, despite soaring new infections. That doesn't mean the virus is less deadly. CNN. 2020 Oct 28. URL: https://www.cnn.com/2020/10/28/europe/ coronavirus-death-rate-second-wave-lower-intl/index.html [accessed 2021-04-09]

62. Data Dashboard - The Global SARS-CoV-2 Surveillance Project (GASSP). Northwestern University. 2021. URL: https:/ /sites.northwestern.edu/covidglobalsurveillance/ [accessed 2021-04-11]

63. Moghnieh R, Abdallah D, Bizri AR. COVID-19: Second wave or multiple peaks, natural herd immunity or vaccine - We should be prepared. Disaster Med Public Health Prep 2020 Sep 10:1-8 [FREE Full text] [doi: 10.1017/dmp.2020.349] [Medline: 32907693] 
64. Lai JW, Cheong KH. Superposition of COVID-19 waves, anticipating a sustained wave, and lessons for the future. Bioessays 2020 Dec;42(12):e2000178 [FREE Full text] [doi: 10.1002/bies.202000178] [Medline: 33040355]

65. Standl F, Joeckel KH, Kowall B, Schmidt B, Stang A. Subsequent waves of viral pandemics, a hint for the future course of the SARS-CoV-2 pandemic. medRxiv. Preprint posted online on July 14, 2020. [FREE Full text] [doi: $10.1101 / 2020.07 .10 .20150698]$

66. Oehmke JF, Oehmke TB, Singh LN, Post LA. Dynamic panel estimate-based health surveillance of SARS-CoV-2 infection rates to inform public health policy: Model development and validation. J Med Internet Res 2020 Sep 22;22(9):e20924 [FREE Full text] [doi: 10.2196/20924] [Medline: $\underline{\text { 32915762] }}$

\author{
Abbreviations \\ EU: European Union \\ GMM: generalized method of moments \\ WB: World Bank \\ WHO: World Health Organization \\ Edited by $G$ Eysenbach; submitted 11.11.20; peer-reviewed by $J$ Walsh; comments to author 03.12.20; revised version received \\ 11.02.21; accepted 04.04.21; published 28.04.21 \\ Please cite as: \\ Post L, Culler K, Moss CB, Murphy RL, Achenbach CJ, Ison MG, Resnick D, Singh LN, White J, Boctor MJ, Welch SB, Oehmke JF \\ Surveillance of the Second Wave of COVID-19 in Europe: Longitudinal Trend Analyses \\ JMIR Public Health Surveill 2021;7(4):e25695 \\ URL: https://publichealth.jmir.org/2021/4/e25695 \\ doi: $10.2196 / 25695$ \\ PMID: 33818391
}

CLori Post, Kasen Culler, Charles B Moss, Robert L Murphy, Chad J Achenbach, Michael G Ison, Danielle Resnick, Lauren Nadya Singh, Janine White, Michael J Boctor, Sarah B Welch, James Francis Oehmke. Originally published in JMIR Public Health and Surveillance (https://publichealth.jmir.org), 28.04.2021. This is an open-access article distributed under the terms of the Creative Commons Attribution License (https://creativecommons.org/licenses/by/4.0/), which permits unrestricted use, distribution, and reproduction in any medium, provided the original work, first published in JMIR Public Health and Surveillance, is properly cited. The complete bibliographic information, a link to the original publication on https://publichealth.jmir.org, as well as this copyright and license information must be included. 